Pacific Journal of Mathematics

MULTIPLICATION FORMULAE FOR PERIODIC FUNCTIONS 


\section{MULTIPLICATION FORMULAE FOR PERIODIC FUNCTIONS}

\section{Herbert Walum}

Carlitz and others have proved that if $f$ is a polynomial such that it satisfies the formula

$$
\sum_{j=0}^{d-1} f\left(x+\frac{j}{d}\right)=d^{1-k} f(d x)
$$

then $f$ is (essentially) the $k^{\text {th }}$ degree Bernoulli polynomial. The purpose of this paper is to discuss the slightly more general formula

$$
\sum_{n(d)} f\left(x+\frac{n}{d}\right)=\theta(d) f(x d) .
$$

when $f$ is periodic with period 1 . The notation $n(d)$ under the summation sign indicates that $n$ runs through a complete system of residues $\bmod d$. Formulae like (1.1) and (1.2) occur also in theories of Franel's formula and in the elementary theory of Dedekind sums.

In this paper we will pretty much characterize the periodic bounded variation solutions of (1.2). Then we provide a weak generalization of the current best form of Franel's theorem, and use this result to provide a method for constructing new solutions of (1.2) from old ones, at least in principle.

1. Introduction. D. H. Lehmer discussed (1.1) and (1.2) at the 1986 West coast number theory conference in Tucson, Arizona and called for examples of (1.1) and (1.2). He gave examples including polynomials, step functions, bounded variation periodic functions, and periodic meromorphic functions.

See $[2,3]$ for discussions of $(1.1)$ and Bernoulli polynomials, [4, $6,8,11$ ] for discussions of Franel's formula and [7] for discussions of Dedekind sums. Since there is some measure of diversity in our theorems, a section will be devoted to each one. Section 2 contains a discussion of two new examples that arise from the fact that its main theorem tells us where not to look for examples of (1.2). Section 4 contains as many examples of (1.2) that are bounded variation that $I$ know of.

2. Periodic bounded variation solutions of (1.2). In this section our bounded variation functions will be identical to their Fourier series. 
One might note that the set of $f$ that satisfies (1.2) is a real vector space. Does (1.2) have non-zero solutions for a given $\theta$ ? The next two simple theorems give some information about this question.

THEOREM 2.1. If $f$ is a non-zero solution of

$$
\sum_{n=0}^{d-1} f\left(x+\frac{n}{d}\right)=\theta(d) f(d x)
$$

then $\theta$ is completely multiplicative.

The earliest form of our proof seems to be due to Carlitz. Note that if $f$ is a solution of $(2.1)$, then

$$
\begin{aligned}
\theta(r s) f(r s x) & =\sum_{j=0}^{r s-1} f\left(x+\frac{j}{r s}\right) \\
& =\sum_{m=0}^{r-1} \sum_{n=0}^{s-1} f\left(x+\frac{m+n r}{r s}\right) \\
& =\sum_{m=0}^{r-1} \sum_{n=0}^{s-1} f\left(x+\frac{m}{r s}+\frac{n}{s}\right) \\
& =\sum_{m=0}^{r-1} \theta(s) f\left(s x+\frac{m}{r}\right) \\
& =\theta(s) \theta(r) f(r s x) .
\end{aligned}
$$

The conclusion $\theta(r s)=\theta(r) \theta(s)$ follows when $x$ is chosen to be a value so that $f(r s x) \neq 0$.

Having noted that $\theta$ must be completely multiplicative for (1.1) or (1.2) or (2.1) to have non-zero solutions, we argue that many formulae (1.2) do have solutions when $\theta$ is completely multiplicative. We use the notation $e(t)=e^{2 \pi i t}$.

THEOREM 2.2. If $\theta$ is completely multiplicative and

$$
f_{\theta}(x)=\sum_{n \neq 0} \frac{\theta(n)}{n} e(n x)
$$


where the series always converges and where the domain of $\theta$ is extended so that $\theta(-n)=-\theta(n)$, then $f_{\theta}$ is a solution of (1.2). The summation in (2.3) is over all non-zero integers.

The proof is easy.

$$
\begin{aligned}
\sum_{j(d)} f_{\theta} & \left(x+\frac{j}{d}\right)=\sum_{j(d)} \sum_{n \neq 0} \frac{\theta(n)}{n} e\left(n\left(x+\frac{j}{d}\right)\right) \\
& =\sum_{n \neq 0} \frac{\theta(n)}{n} e(n x) \sum_{j(d)} e\left(\frac{n j}{d}\right) \\
& =\sum_{\substack{n \neq 0 \\
d \mid n}} d \frac{\theta(n)}{n} e(n x) \\
& =\sum_{m \neq 0} d \frac{\theta(m d)}{m d} e(m d x) \\
& =\theta(d) \sum_{m \neq 0} \frac{\theta(m) e(m d x)}{m}=\theta(d) f(d x) .
\end{aligned}
$$

Theorem 2.1 suggests asking for solutions of (1.2) when $\theta$ is a familiar completely multiplicative function, for example $\theta$ is a Dirichlet character or given by the formula $\theta(d)=d^{n}$ or the Liouville- $\lambda$ function. Theorem 2.3 gives a solution in case $\theta$ is a Dirichlet character, but in case $\theta$ is $\lambda$, Theorem 2.2 gives information only at some cost. For example, the convergence of the series for $f_{\lambda}$ is not trivial. This example will be discussed in greater detail in $\S 4.3$.

What kind of converse of Theorem 2.2 can we arrange?

THEOREM 2.3. If $f$ is (a) periodic with period 1 and satisfies (1.2) with a completely multiplicative $\theta$, (b) real and (c) bounded variation and identical to its Fourier series, then $f=c f_{\theta}$ where $c$ is a constant.

This result is also easy to prove. Since $f$ is bounded variation, period 1 we can write

$$
f(x)=\sum_{n} a_{n} e(n x)
$$

for all values of $x$. Then, using (1.2), the series for $x$, interchanging summations and finally using the formula for the sum of a geometric 


\section{progression}

$$
\begin{aligned}
\sum_{m} \theta(d) a_{m} e(m d x)=\theta(d) f(d x) \\
=\sum_{j(d)} f\left(x+\frac{j}{d}\right)=\sum_{j(d)} \sum_{n} a_{n} e\left(n\left(x+\frac{j}{d}\right)\right) \\
=\sum_{n} a_{n} e(n x) \sum_{j(d)} e\left(\frac{j n}{d}\right)=\sum_{d \mid n} d a_{n} e(n x) \\
=\sum_{m} d a_{m d} e(m d x) .
\end{aligned}
$$

By the identity theorem for Fourier series, $\theta(d) a_{m}=d a_{m d}$ for all $m$ and $d$. Taking $m=1$ gives $a_{d}=a_{1} \frac{\theta(d)}{d}$. Noting that since $f$ is real, $a_{-d}=d_{d}$ the result follows.

Where else would one look for solutions of (1.2)? We offer two suggesions.

First, letting $Q$ and $R$ be the rationals and reals as usual, let $V$ be a rational vector space such that $Q \subset V \subset R$. Let $\rho_{V}(v)=1$ when $v \in V$ and $p_{V}(v)=0$ when $v \notin V$. Now $x, x+\frac{j}{d}, d x$ (where $j$ is in a complete residue system $\bmod d$ ) are all in $V$ or none are in $V$. From this fact $p_{V}(x) f(x)$ is a solution of (1.2) when $f$ is a solution of (1.2).

Next, let $F$ be a real arithmetic function such that $F(d n, d r)=$ $F(n, r)$ and $F(n+r, r)=F(n, r)$. Define $f(p / q)=F(p, q)$. It is easy to see that

$$
\sum_{j(d)} F(n+j d, r d)=\theta(d) F(n, r)
$$

is sufficient to derive (1.2) for $f$. I would think that characterizing the set of $F$ that satisfies the above three conditions would be interesting. I offer one class of examples for $F$ as just described. Let $P$ be a set of primes and let $A$ be the set of all natural numbers all of whose prime factors are in $P$. Define $\gamma_{P}(n)$ to be the largest factor of $n$ that is in $A$, and let $F_{P}(x, r)$ be 1 if $\gamma_{P}(r)$ divides $x$ and 0 otherwise. Since $\gamma_{P}(r) \mid r$, it follows that $\gamma_{P}(r) \mid x$ is equivalent to $\gamma_{P}(r) \mid x+r$ and hence $F_{P}(x+r, r)=F_{P}(x, r)$. It is also clear that $\gamma_{P}$ is completely multiplicative and that $\gamma_{P}(d r) \mid d x$ is equivalent to $\gamma_{P}(r) \mid x$. Hence, $F_{P}(d x, d r)=F_{P}(x, r)$. Finally,

$$
\begin{aligned}
1 & =F_{P}(x+j r, d r) \Leftrightarrow \gamma_{P}(d r)\left|x+j r \Leftrightarrow \gamma_{P}(d) \gamma_{P}(r)\right| x+j r \\
& \Rightarrow \gamma_{P}(r) \mid x \Rightarrow F(x, r)=1 .
\end{aligned}
$$


Thus, there is a $j$ so that $F_{P}(x+j r, d r)=1$ only when $f_{P}(x, r)=1$. Thus to prove (2.6) we only need consider the case $F_{P}(x, r)=1$ since $F_{P}(x, r)=0$ implies that all the terms in (2.6) are 0 . Thus, assume $F_{P}(x, r)=1$ or that $\gamma_{P}(r) \mid x$. Then

(2.7) $1=F_{P}(x+j r, d r) \Leftrightarrow j\left(r / \gamma_{P}(r)\right) \equiv\left(-x / \gamma_{P}(r)\right)\left(\bmod \gamma_{P}(d)\right)$.

This last congruence has exactly $d \gamma_{P}(d)$ solutions as $j$ runs through a complete system of residue $\bmod d$. It follows that $F_{P}$ satisfies $(2.6)$ with $\theta(d)=d / \gamma_{P}(d)$, i.e., $\theta(d)$ is the part of $d$ that is made from prime factors of $d$ where the primes are not in $P$.

3. Franel's formula. Franel's formula is

$$
\int_{0}^{1} \psi(m t) \psi(n t)=\frac{(m, n)^{2}}{12 m n}
$$

where $\psi(t)=t-[t]-\frac{1}{2}$. It was made famous by Landau in his "Vorlesungen über die Zahlentheorie," Mikolas $[9,10]$ has offered various generalizations of (3.1), and here we prove, using standard methods,

THEOREM 3.1. Let $f$ and $g$ be periodic with period 1 and satisfy

$$
\begin{gathered}
\sum_{j(d)} f\left(x+\frac{j}{d}\right)=\theta_{f}(d) f(x d), \\
\sum_{j(d)} g\left(x+\frac{j}{d}\right)=\theta_{g}(d) g(x d)
\end{gathered}
$$

for all $d$ and $x$ so that the quantities $x+\frac{j}{d}$ and $x d$ are in the domains of $f$ and $g$. Then if the integral on the left-hand side of

$$
\begin{array}{rl}
\int_{0}^{1} & f(\alpha+m t) g(\beta+n t) d t \\
= & \frac{(m, n)^{2}}{m n} \theta_{f}\left(\frac{n}{(m, n)}\right) \theta_{g}\left(\frac{m}{(m, n)}\right) \\
& \cdot \int_{0}^{1} f\left(\frac{n \alpha}{(m, n)}+t\right) g\left(\frac{m \beta}{(m, n)}+t\right) d t
\end{array}
$$

exists for $m$ and $n$ integers and $\alpha$ and $\beta$ real numbers, the integral on the right-hand side of (3.3) exists and (3.3) is correct.

The integrals in (3.3) must satisfy the following propertie;

$$
\int_{a}^{b}\left[c_{1} f_{1}(t)+c_{2} f_{2}(t)\right] d t=c_{1} \int_{a}^{b} f_{1}(t) d t+c_{2} \int_{a}^{b} f_{2}(t) d t
$$




$$
\int_{l(a)}^{l(b)} f(t) d t=\int_{a}^{b} f(l(x)) l^{1}(x) d x,
$$

where $l(x)=c x+d$ and

$$
\int_{a}^{b} f(t) d t=\int_{a}^{c} f(t) d t+\int_{c}^{d} f(t) d t
$$

so long as all the integrals exist. In case the integrals are Cauchy mean values (we will use Cauchy mean values in $\S 4$ ) (3.13) is false, but Theorem 3.1 is true so long as $p \alpha+q$ and $p \beta+q$ are finite points of $f$ and $g$ respectively where $p$ and $q$ are integers.

The proof uses familiar principles. We first prove under the hypothesis of the theorem that the formulae

$$
\begin{aligned}
& \int_{0}^{1} f(\alpha+m t) g(\beta+n t) d t \\
& \quad=\frac{(m, n)}{m} \theta_{g}\left(\frac{m}{(m, n)}\right) \int_{0}^{1} g\left(\frac{m \beta}{(m, n)} t\right) f(\alpha+t) d t
\end{aligned}
$$

is true for all integers $m$ and $n$. The integral on the right-hand side of (3.4) has the same form as the integral on the left-hand side of (3.4). Applying (3.4) to itself, so to speak, will give the Theorem. Let $d=(m, n), m=d M, n=d N$. Then

$$
\begin{aligned}
\int_{0}^{1} f(\alpha+m t) g(\beta+n t) d t=\frac{1}{m} \int_{0}^{m} f(\alpha+t) g\left(\beta+\frac{n}{m} t\right) d t \\
\quad=\frac{1}{m} \sum_{j=0}^{m-1} \int_{j}^{j+1} f(\alpha+t) g\left(\beta+\frac{N}{M} t\right) d t \\
=\frac{1}{m} \int_{0}^{1} f(\alpha+t) \sum_{j=0}^{m-1} g\left(\beta+\frac{N}{M}(t+j)\right) d t \\
=\frac{1}{m} \int_{0}^{1} f(\alpha+t) \sum_{u=0}^{d-1} \sum_{v=0}^{M-1} g\left(\beta+\frac{N t}{M}+\frac{N}{M}(v+u M)\right) d t \\
=\frac{d}{m} \int_{0}^{1} f(\alpha+t) \sum_{N(M)} g\left(\beta+\frac{N t}{M}+\frac{N v}{M}\right) d t .
\end{aligned}
$$

Since $(N, M)=1$, as $N$ runs through a complete system of residues $\bmod m$ so does $N v$. Thus, we may replace $N v$ by $v$ and obtain for 
the left-hand side of the above expression

$$
\begin{array}{rl}
\frac{d}{m} \int_{0}^{1} & f(\alpha+t) \sum_{v(M)} g\left(\beta+\frac{N t}{M}+\frac{v}{M}\right) d t \\
& =\frac{d}{m} \int_{0}^{1} f(\alpha+t) \theta_{g}(M) g(M \beta+N t) d t
\end{array}
$$

and (3.4) is deduced.

4. The construction of new examples from old. Parseval type formulae. Examples. Let $f$ and $g$ satisfy

$$
f(x)=\sum_{m \neq 0} \frac{\theta_{f}(m)}{m} e(m x), \quad g(x)=\sum_{n \neq 0} \frac{\theta_{g}(n)}{n} e(n x),
$$

where we temporarily assume the series are absolutely convergent. If we form

$$
h(x)=\int_{0}^{1} f(x+t) g(t) d t
$$

then $h(x)$ is equal to

$$
\begin{aligned}
\int_{0}^{1} & \sum_{m \neq 0} \frac{\theta_{f}(m)}{m} e(m(x+t)) \sum_{n \neq 0} \frac{\theta_{g}(n)}{n} e(n t) d t \\
& =\sum_{m, n \neq 0} \frac{\theta_{f}(m) \theta_{g}(n)}{m n} e(m x) \int_{0}^{1} e((n+m) t) d t \\
& =\sum_{m \neq 0} \frac{\left(\theta_{f}(m) \theta_{g}(-m) /(-m)\right)}{m} e(m x)
\end{aligned}
$$

and $h(x)$ will satisfy (1.2) with

$$
\theta(d)=\theta_{f}(d) \theta_{g}(d) / d .
$$

Theorem 4.1 will obtain the same conclusions on weaker hypotheses and will be deduced from Theorem 3.1 instead of Fourier analysis.

THEOREM 4.1. Let $f$ and $g$ be periodic with period 1 and satisfy (3.2). Let $h$ be defined by equation (4.2). Then $h$ satisfies (1.2) with (4.3) being true.

From (4.2) we obtain

$$
\begin{aligned}
\sum_{j(d)} h\left(x+\frac{j}{d}\right) & =\int_{0}^{1} g(t) \sum_{j(d)} f\left(x+t+\frac{j}{d}\right) d t \\
& =\theta_{f}(d) \int_{0}^{1} g(t) f(d x+d t) d t
\end{aligned}
$$


By Theorem (3.1), the left-hand side of the above formula is

$$
\theta_{f}(d) \theta_{f}\left(\frac{1}{(1, d)}\right) \theta_{g}\left(\frac{d}{(1, d)}\right) \frac{(1, d)^{2}}{1 \cdot d} \int_{0}^{1} f\left(d x \cdot \frac{1}{(1, d)}+t\right) g(0+t) d t
$$

and the result follows so long as the integration method satisfies (3.1.1), (3.1.2) and (3.1.3). If the integrals are Cauchy mean values, then we must assume that $p x+q$ is a finite point of $f$ and $g$ when $p$ and $q$ are integers.

We now proceed to a discussion of examples of (1.2).

In order to illustrate the above theorems, we will discuss examples of solutions of (1.2) in case $\theta(d)=d^{n}, \theta(d)=\chi(d) d^{n}$ and $\theta(d)=\lambda(d) d^{n}$. In these examples, $\chi$ is a Dirichlet character, and $\lambda$ is Lioville's function, i.e., $\lambda(p)=-1$ for $p$ a prime, and $\lambda$ is completely multiplicative. Thus, we will have discussed (1.2) in the cases when $\theta$ is one of the common completely multiplicative functions. In the case $\theta(d)=d^{n}$ and $\theta(d)=\chi(d) d^{n}$ we will encounter familiar examples, when $\theta(d)=\lambda(d) d^{n}$, less so.

4.1. $\theta(d)=d^{n}$.

Since the series

$$
\psi_{m}(x)=(2 \pi i)^{-m} \sum_{k \neq 0} k^{-m} e(n k)
$$

converges for $m \geq 1$ and differs from the periodic extension of the Bernoulli polynomial of degree $m$ by a constant factor, we have an example with $\theta(d)=d^{n}$ when $n=1-m$ or when $n$ is not a positive integer. In fact, when $n=0, m=1, \psi_{1}(x)=((x))$, a familiar function from the theory of Dedekind sums [7, p.1]. This same Carus monograph just referred to also provides an example for $n$ a positive integer. In fact, in the second variation of the third proof of the reciprocity formula [see (27) page 18 of 7] for Dedekind sums, Rademacher-Grosswald give $(x=k y)$

$$
\sum_{m=1}^{k} \cot \left(y+\frac{m}{k}\right) \pi=k \cot k y \pi
$$

and thus $\cot y \pi$ is an example of (1.2) with $n=1$. Since $\cot \pi y$ is meromorphic one can easily see that $\pi^{1-n} d^{n-1} / d y^{n-1} \cot \pi y$ is a solution when $n \geq 2$. It can easily be directly verified that

$$
M_{n}(z)=\sum_{n} \frac{(-1)^{n}(n !)}{(z+m)^{n}}=\frac{d^{n-1}}{d x^{n}} \pi \cot \pi z
$$

is also a solution when $n \geq 1$ (see [10] alsc). 
I know of no other periodic meromorphic solutions of (1.2) when $n \geq 1$. Of course, there can be no bounded variation periodic solutions of (1.2) for $n \geq 1$, if these solutions are exactly equal to their Fourier series, by Theorem 2.2. Naturally if $\rho_{V}$ is the vector space characteristic function from $\S 2, \rho_{V}(x) \psi_{m}(x)$ and $\rho_{V}(x) M_{n}(z)$ provide infinitely many solutions to (1.2) in the case $\theta(d)=d^{n}$. Naturally, this last class of examples cannot be bounded variation.

$$
\theta(d)=\chi(d) d^{n}
$$

This section could be considered to be a generalization of 4.1. Here, $\chi$ is a Dirichlet character. Define

$$
\psi(\chi, m, x)=(2 \pi i)^{-m} \sum_{j \neq 0} \chi(j) j^{-m} e(j x) .
$$

If $r$ is the modulus of $\chi$, then

$$
\psi(\chi, m, x)=\sum_{k(r)} \chi(k)(2 \pi i)^{-m} \sum_{\substack{j \equiv k(r) \\ j \neq 0}} j^{-m} e(j x) .
$$

Next, consider

$$
\begin{aligned}
\frac{1}{r} \sum_{s(r)} \psi_{m}\left(x+\frac{s}{r}\right) e\left(\frac{-s k}{r}\right) \\
=(2 \pi i)^{-m} \sum_{j \neq 0} j^{-m}\left(\frac{1}{r} \sum_{s(r)} e\left(j\left(x+\frac{s}{r}\right)-\frac{s k}{r}\right)\right) \\
=(2 \pi i)^{-m} \sum_{j \neq 0} j^{-m} e(j x)\left(\frac{1}{r} \sum_{s(r)} e\left(\frac{s(j-k)}{r}\right)\right) \\
=(2 \pi i)^{-m} \sum_{\substack{j \equiv k(r) \\
j \neq 0}} j^{-m} e(j x) .
\end{aligned}
$$

Thus, if we combine (4.2.3) and (4.2.2) we obtain

$$
\begin{aligned}
\psi(\chi, m, x) & =\frac{1}{r} \sum_{k(r)} \sum_{s(r)} \chi(k) e\left(\frac{-s k}{r}\right) \psi_{m}\left(x+\frac{s}{r}\right) \\
& =\frac{1}{r} \sum_{s(r)} \psi_{m}\left(x+\frac{s}{r}\right) \sum_{k(r)} \chi(k) e\left(\frac{-s k}{r}\right) \\
& =\frac{1}{r} \sum_{s(r)} \psi_{m}\left(x+\frac{s}{r}\right) G_{-s}(\chi),
\end{aligned}
$$


where $G_{t}(\chi)$ is the Gauss sum

$$
G_{t}(\chi)=\sum_{k(r)} \chi(k) e\left(\frac{t k}{r}\right) .
$$

Thus, we have expressed $\psi(\chi, m, x)$ in terms of more familiar functions. The reader might compare (4.2.4) to (4.9) of [9]. Also note Proposition 9.7 of that same paper, and also the elegant formulae of $\S 10$.

From (4.2.1) it is obvious that $\psi(\chi, m, x)$ is a solution of (1.2) with $\theta(d)=\chi(d) d^{1-n}$ for $n \geq 1$. We use Theorem 4.1 to construct solutions of (1.2) with $\theta(d)=\chi(d) d^{n}$ for $n$ positive. Consider

$$
f(x)=\int_{0}^{1} \psi(\chi, 1, t) M_{3}(t+x) d t
$$

by Theorem 4.1, $f$ satisfies (1.2) with $\theta(d)=\chi(d) d^{3} / d=d^{2} \chi(d)$ if the integral (4.2.6) converges. We will see that (4.2.6) converges as a Cauchy mean value in the process of computing (4.2.6).

We start with the indefinite integral, for $n$, a positive integer,

$$
\int\left(t-\frac{1}{2}\right) M_{n+2}(x+t) d t=\left(t-\frac{1}{2}\right) M_{n+1}(x+t)-M_{n}(x+t)
$$

which is valid for $t$ in any interval not containing $m-x$ for $m$ an integer. Taking $x \in(0,1)$,

$$
\begin{gathered}
\int_{0}^{1-x-\varepsilon} \psi_{1}(t) M_{n+2}(x+t) d t+\int_{1-x+\varepsilon}^{1} \psi(t) M_{n+2}(x+t) d t \\
=M_{n+1}(x)+\left(\frac{1}{2}-x\right)\left(M_{n+1}(-\varepsilon)-M_{n+1}(\varepsilon)\right) \\
-\varepsilon\left(M_{n+1}(-\varepsilon)+M_{n+1}(\varepsilon)\right)+\left(M_{n}(\varepsilon)-M_{n}(-\varepsilon)\right) .
\end{gathered}
$$

The limit, as $\varepsilon$ tends to zero in (4.2.7) exists when $n$ is odd. For odd $n$, the Cauchy mean value

$$
\int_{0}^{1} \psi_{1}(t) M_{n+2}(t+x)
$$

is

$$
M_{n+1}(x)+\lim _{\varepsilon \rightarrow 0}\left(2 M_{n}(\varepsilon)-2 \varepsilon M_{n+1}(\varepsilon)\right) .
$$

By (4.1.3), the limit is $-4 n ! \zeta(n)$ and hence,

$$
\int_{0}^{1} \psi_{1}(t) M_{n+2}(x+t) d t=M_{n+1}(x)-4 n ! \zeta(n)
$$

for $n$ odd and positive. 
Keeping $n$ odd and positive, and working backwards from a formal calculation,

$$
\text { (4.2.9) } \begin{aligned}
\frac{1}{r} \sum_{s(r)} & G_{s}(\chi) M_{n+1}\left(x+\frac{s}{r}\right) \\
& =\frac{1}{r} \sum_{s(r)} G_{s}(\chi)\left(M_{n+1}\left(x-\frac{s}{r}\right)-4 n ! \zeta(n)\right) \\
& =\frac{1}{r} \sum_{s(r)} G_{x}(\chi) \int_{0}^{1} \psi_{1}(t) M_{n+2}\left(t+x-\frac{s}{r}\right) d t \\
& =\frac{1}{r} \sum_{s(r)} G_{x}(\chi) \int_{0}^{1} \psi_{1}\left(t+\frac{s}{r}\right) M_{n+2}(t+x) d t \\
& =\int_{0}^{1} \psi(\chi, 1, t) M_{n+2}(t+x) d t \stackrel{\text { def }}{=} M(\chi, n+1, x) .
\end{aligned}
$$

By (4.2.8), this last integral exists as a Cauchy mean value and by Theorem 4.1 satisfies (1.2) with

$$
\theta(d)=\chi(d) d^{n+2} / d=\chi(d) d^{n+1} .
$$

Thus, we have an example of (1.2) with $\theta(d)=\chi(d) d^{n}$ for all integers $n$ except negative odd integers. Differentiating $M(\chi, n, x)$ when $n$ is a negative even integer gives examples of $(1.2)$ with $\theta(d)=$ $\chi(d) d^{n+1}$. Thus, we have examples for all exponents except -1 . Presumably $M(\chi, 1, x)$ should do the job, but the present theorems only suggest that it does.

A word here about the domain of $M(\chi, n+1, x)=M$. By (4.2.9), the domain of $M$ is the reals (or complexes) excluding the integers. Thus, $M(\chi, n, x)$ satisfies (1.2) for all $d$ and some $x$. Perhaps, however, they are the best, or only, meromorphic solutions of (1.2).

$$
\theta(d)=-\lambda(d) d^{n}
$$

By Theorem 2.1,

$$
L_{n}(x)=\sum_{k \neq 0} \frac{\lambda(k)}{k^{n+1}} e(k x)
$$

satisfies (1.2) with $\theta(d)=\lambda(d) d^{-n}$ for $n \geq 1$. The convergence domain for (4.3.1) when $n=0$ seems unknown, although it follows from a theorem of Davenport's. 
The theorem of Davenport [5] is

THEOREM 4.3.1. If $k$ is a positive number, and $t$ is real, then

$$
\sum_{n \leq x} \mu(n) e(n t)=O\left(x \log ^{-k} x\right)
$$

uniformly in $t$ as $x$ tends to infinity.

A standard lattice point argument enables us to deduce the next theorem from (4.3.2).

THEOREM 4.3.2. If $k$ is a positive number and $t$ is real, then

$$
\sum_{n \leq x} \lambda(n) e(n t)=O\left(x \log ^{k} x\right)
$$

uniformly in $t$ as $x$ tends to infinity.

Now, since

$$
\lambda(n)=\sum_{u^{2} v=n} \mu(v)
$$

we have

$$
\sum_{n \leq x} \lambda(n) e(n t)=\sum_{u^{2} v \leq x} \mu(v) e\left(u^{2} v t\right)
$$

The sum on the right-hand side of (4.3.5) may be regarded as a sum over the lattice points satisfying $1 \leq u, 1 \leq v, u^{2} v \leq x$. This region may be broken into three parts by a point $(H, K)$ on $u^{2} v=x$ in the standard way one finds in elementary theories of the divisor problem. One obtains

$$
\begin{aligned}
\sum_{n \leq x} \lambda(n) e(n t)= & \sum_{u \leq H} \sum_{v \leq x / u^{2}} \mu(v) e\left(v\left(u^{2} t\right)\right) \\
& +\sum_{v \leq K} \mu(v) \sum_{u \leq \sqrt{x / v}} e\left(u^{2}(v t)\right) \\
& -\sum_{\substack{u \leq H \\
v \leq K}} \mu(v) e\left(u^{2} v t\right)=S_{1}+S_{2}-S_{3} .
\end{aligned}
$$


The terms in these sums are one in absolute value, so $S_{2}$ and $S_{3}$ may be estimated by their number of terms. Thus $S_{2}=O(\sqrt{x K})$ and $S_{3}=O(H K)$. Also,

$$
S_{1}=\sum_{u \leq H} O\left(\frac{x}{u^{2}\left(\log \left(x / u^{2}\right)\right)^{k}}\right)=O\left(\frac{x}{(\log x)^{k}}\right)
$$

if we take $H=x^{1 / 4}, K=\sqrt{x}$ and thus we obtain (4.3.3).

Now partial summation applied to

$$
\sum_{n=P}^{Q} \frac{\lambda(n)}{n} e(n t)
$$

using (4.3.3) gives (4.3.8) to be $\left.O(P / \log P)^{K-1}\right)$ and thus by standard theorems in the theory of uniform convergence

$$
f_{\lambda}(t)=\sum_{n \neq 0} \frac{\lambda(n)}{n} e(n t)
$$

is uniformly convergent and bounded for all $t$. Thus, also $g_{\lambda}$ is bounded and continuous.

\section{REFERENCES}

[1] B. C. Berendt and L. Schoenfeld, Periodic analogues of the Euler-Maclaurin and Poisson Summation formulas with applications to number theory, Acta Arith., 28 (1975), 23-68.

[2] L. Carlitz, The multiplication formulas for the Bernoulli and Euler polynomials, Math. Mag., 27 (1952), 59-64.

[3] _ A note on the multiplication formulas for the Bernoulli and Euler polynomials. Proc. Amer. Math. Soc., 4 (1953), 184-188.

[4] _ Some finite summation formulas of arithmetic character, Publ. Math. Debrecen, 6 (1959), 262-268.

[5] H. Davenport, Collected Works, Academic Press, New York. Vol. IV No. 2.

[6] J. Franel, Les suites de Farey et le problème des nombres premiers, Nachr. Ges. Wiss. Göttingen, (1924), 198-201.

[7] E. Grosswald and H. Rademacher, Dedekind Sums, Amer. Math. Soc. Carus Monographs, No. 16.

[8] E. Landau, Bemerkungen zu der vorstehenden Abhandlung von Herrn Franel, Nachr. Ges. Wiss. Göttingen, (1924), 202-206.

[9] M. Mikolás, Integral formulae of arithmetical characteristics relating to the zetafunction of Hurwitz, Publ. Math. Debrecen, 5 (1957), 44-53. 
[10] M. Mikolás, Mellinsche Transformation und Orthogonalität bei $\zeta(s, u)$. Verallgemeinerung der Riemannschen Funktionalgleichung von $\zeta(s)$, Acta Sci. Math. (Szeged), 17 (1956), 143-164.

[11] L. J. Mordell, Integral formulae of arithmetical character, J. London Math. Soc., 33 (1958), 371-375.

Received June 13, 1988, and in revised form January 25, 1989.

Ohio State University

Columbus, OH 43210-1174 


\section{PACIFIC JOURNAL OF MATHEMATICS EDITORS}

\author{
V. S. VARAdaraJan \\ (Managing Editor) \\ University of California \\ Los Angeles, CA 90024-1555-05 \\ Herbert Clemens \\ University of Utah \\ Salt Lake City, UT 84112 \\ THOMAS ENRIGHT \\ University of California, San Diego \\ La Jolla, CA 92093
}

R. FINN

Stanford University

Stanford, CA 94305

Hermann FlaschKa

University of Arizona

Tucson, AZ 85721

VAUGHAN F. R. Jones

University of California

Berkeley, CA 94720

STEVEN KerckHofF

Stanford University

Stanford, CA 94305

\section{C. MOORE}

University of California

Berkeley, CA 94720

Martin ScharlemanN

University of California

Santa Barbara, CA 93106

HAROLd STARK

University of California, San Diego

La Jolla, CA 92093
R. ARENS
E. F. BECKENBACH
F. WoLF
K. YoshidA
ASSOCIATE EDITORS

\begin{tabular}{ll}
\multicolumn{1}{c}{$(1906-1982)$} & \multicolumn{1}{c}{$(1904-1989)$} \\
SUPPORTING & INSTITUTIONS \\
UNIVERSITY OF ARIZONA & UNIVERSITY OF OREGON \\
UNIVERSITY OF BRITISH COLUMBIA & UNIVERSITY OF SOUTHERN CALIFORNIA \\
CALIFORNIA INSTITUTE OF TECHNOLOGY & STANFORD UNIVERSITY \\
UNIVERSITY OF CALIFORNIA & UNIVERSITY OF HAWAII \\
MONTANA STATE UNIVERSITY & UNIVERSITY OF TOKYO \\
UNIVERSITY OF NEVADA, RENO & UNIVERSITY OF UTAH \\
NEW MEXICO STATE UNIVERSITY & WASHINGTON STATE UNIVERSITY \\
OREGON STATE UNIVERSITY & UNIVERSITY OF WASHINGTON
\end{tabular}

The Supporting Institutions listed above contribute to the cost of publication of this Journal, but they are not owners or publishers and have no responsibility for its content or policies.

Mathematical papers intended for publication in the Pacific Journal of Mathematics should be in typed form or offset-reproduced (not dittoed), double spaced with large margins. Please do not use built up fractions in the text of the manuscript. However, you may use them in the displayed equations. Underline Greek letters in red, German in green, and script in blue. The first paragraph must be capable of being used separately as a synopsis of the entire paper. In particular it should contain no bibliographic references. Please propose a heading for the odd numbered pages of less than 35 characters. Manuscripts, in triplicate, may be sent to any one of the editors. Please classify according to the 1991 Mathematics Subject Classification scheme which can be found in the December index volumes of Mathematical Reviews. Supply name and address of author to whom proofs should be sent. All other communications should be addressed to the managing editor, or Elaine Barth, University of California, Los Angeles, California 90024-1555-05.

There are page-charges associated with articles appearing in the Pacific Journal of Mathematics. These charges are expected to be paid by the author's University, Government Agency or Company. If the author or authors do not have access to such Institutional support these charges are waived. Single authors will receive 50 free reprints; joint authors will receive a total of 100 free reprints. Additional copies may be obtained at cost in multiples of 50 .

The Pacific Journal of Mathematics (ISSN 0030-8730) is published monthly except for July and August. Regular subscription rate: $\$ 190.00$ a year (10 issues). Special rate: $\$ 95.00$ a year to individual members of supporting institutions.

Subscriptions, orders for numbers issued in the last three calendar years, and changes of address should be sent to Pacific Journal of Mathematics, P.O. Box 969, Carmel Valley, CA 93924, U.S.A. Old back numbers obtainable from Kraus Periodicals Co., Route 100, Millwood, NY 10546.

The Pacific Journal of Mathematics at P.O. Box 969, Carmel Valley, CA 93924 (ISSN 0030-8730) is published monthly except for July and August. Second-class postage paid at Carmel Valley, California 93924, and additional mailing offices. Postmaster: send address changes to Pacific Journal of Mathematics, P.O. Box 969, Carmel Valley, CA 93924.

PUBLISHED BY PACIFIC JOURNAL OF MATHEMATICS, A NON-PROFIT CORPORATION Copyright (C) 1991 by Pacific Journal of Mathematics 


\section{Pacific Journal of Mathematics}

Vol. 149, No. $2 \quad$ June, 1991

Manuel Alfaro Garcia, Mark Conger and Kenneth Hodges, The structure

of singularities in $\Phi$-minimizing networks in $\mathbf{R}^{2}$

Werner Balser, Dependence of differential equations upon parameters in

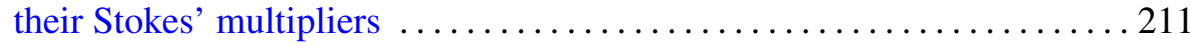

Enrico Casadio Tarabusi and Stefano Trapani, Envelopes of holomorphy

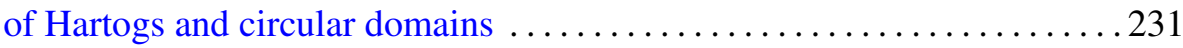

Hermann Flaschka and Luc Haine, Torus orbits in $G / P \ldots \ldots \ldots \ldots 251$

Gyo Taek Jin, The Cochran sequences of semi-boundary links .......... 293

Yasuyuki Kawahigashi, Cohomology of actions of discrete groups on

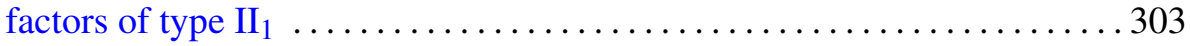

Ki Hyoung Ko and Lawrence Smolinsky, A combinatorial matrix in 3-manifold theory

W. B. Raymond Lickorish, Invariants for 3-manifolds from the combinatorics of the Jones polynomial

Peter Arnold Linnell, Zero divisors and group von Neumann algebras . . . . . 349

Bruce Harvey Wagner, Classification of essential commutants of abelian

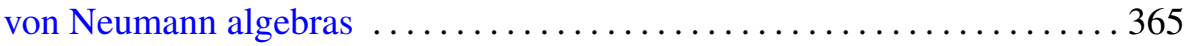

Herbert Walum, Multiplication formulae for periodic functions ..........383 